\title{
An assessment of medication safety practices in selected public health facilities in Kaduna State, Nigeria
}

\author{
Basira Kankia LAWAL ${ }^{1,4 *}$, Aliyu A ALHAJI'2, Bilkisu Bello MAIHA ${ }^{3}$ and Shafiu \\ MOHAMMED ${ }^{4,5}$ \\ ${ }^{l}$ Department of Clinical Pharmacy and Pharmacy Management, Kaduna State University, Kaduna. Nigeria. \\ ${ }^{2}$ Department of Community Medicine; ${ }^{3}$ Department of Pharmacology and Therapeutics; ${ }^{4}$ Department of Clinical \\ Pharmacy and Pharmacy Policy; ${ }^{5}$ Health Systems and Policy Research Unit Ahmadu Bello University, Zaria.
} Nigeria.

Received $14^{\text {th }}$ January 2020; Accepted $28^{\text {th }}$ February 2020

\begin{abstract}
Medication errors occur frequently and have significant clinical and financial consequences, which could be preventable. Unsafe medication practices and errors have been found to be amongst the leading cause of injury and avoidable harm in both developed and developing countries. The World Health Organisation (WHO) has recommended implementation of basic solutions to improve medication safety. This study assessed the presence of basic medication safety practices in four (4) public health facilities in Kaduna State, Nigeria. A validated survey tool was adopted which contains sections that assessed practices such as look-alike, sound-alike (LASA) medications, transitions in care, use of information technology, drug information and other practices. A structured interview was conducted with each head of Pharmacy department of the facilities after obtaining their consent. Only one facility had a medication safety committee, none of the facilities had a list of LASA medications nor a list of error prone abbreviations. Only one facility involved pharmacists in obtaining medication histories and none of the facilities uses 'high risk' warning label on diluted electrolyte solutions. Basic practices to improve medication safety were not implemented in the facilities. Effort must be put in place for the adoption of medication safety practices. Regulations and policies need to be implemented regarding these practices.
\end{abstract}

Keywords: Medication safety; Health facilities; Kaduna State; Nigeria; Medication errors

\section{INTRODUCTION}

Medication errors are the most common type of medical errors and are associated with considerable healthcare expenses [1]. Medication errors occur frequently and have significant clinical and financial consequences, which could be preventable [2]. Research has shown that medical errors and the associated injuries are a significant problem [3]. Additionally, unsafe medication practices and errors have been found to be amongst the leading cause of injury and avoidable harm in both developed and developing countries [4]. It is well established that inappropriate medication use, unsafe medication practices, as well as medication errors continually present a negative impact on patient and economic outcomes all over the world [5]. It is of concern that most harm caused by medicines

\footnotetext{
*Correspondence. E-mail: lawalbasira@yahoo.com Tel: +234-8036063257.

ISSN 0189-8442

2020 Published by Faculty of Pharmaceutical Sciences, University of Jos, Nigeria. Under Creative Commons Attribution-NonCommercial 4.0 International License. https://creativecommons.org/licenses/by-nc/4.0/
} 
is preventable and as such, this issue is currently being given priority in various sectors, including health policy and academia. The World Health Organisation (WHO) further explains that medication safety can be used to describe systematic assessments of healthcare professionals' practices as related to safe use of medicines [5]. Identification and prevention of medication errors is the key component of medication safety. This includes multiple aspects of medication practice and other factors which affect it such as, organizational structure, communication, technologies such as those used for dispensing, and strategies pursued by leadership in cultivating and promoting a culture of safety.

Over the previous years, research has shown that medication errors could be decreased by many interventions. It has been reported that research has shown medication errors could be decreased by many interventions, which in the developed nations include mostly the use of information technology and automation [6]. Other methods include involving a pharmacist with the medical team and prevention of adverse drug events (ADEs) through the application of some core practices [7-9].

In 2005, the WHO launched the World Alliance for Patient Safety and by 2007, the Alliance recommended patient safety solutions to help prevent medication errors and adverse events [10]. These patient safety solutions are standardised tools for healthcare professionals to prevent potential errors from reaching the patient. The patient safety solutions were developed through the WHO Collaborating Centre for Patient Safety Solutions through the activities of the Joint Commission and the Joint Commission International (JCI). The Solutions developed are intended to promote an environment and support systems that minimize the risk of harm despite the complexity and lack of standardization in modern health care. The term "Patient Safety Solution" is thus, defined as "Any system design or intervention that has demonstrated the ability to prevent or mitigate patient harm stemming from the process of health care" [10]. The Solutions include topics on: look-alike, sound-alike (LASA) medication names; patient identification; communication during patient handovers; performance of correct procedure at correct body site; control of concentrated electrolyte solutions; assuring medication accuracy at transitions in care; avoiding catheter and tubing misconnections; single use of injection devices and; improved hand hygiene to prevent healthcare associated infection [10].

No study has been conducted in Kaduna State, or even in Nigeria to assess medication safety practices in public health facilities. Thus, it is important to understand the present status of practices pertaining to medication safety so as to put in place ways to guide healthcare professionals, healthcare organisations, health ministries and policy makers on areas for improvement. This study aims to assess the presence of medication safety practices in public health facilities in Kaduna State, Nigeria.

\section{METHODS}

Study design. The study was conducted as a structured interview based study.

Study setting. Kaduna State is the third most populous state in Nigeria with an estimated population of 8.6 million people as at 2018 . There are 23 Local Government Areas and 255 wards. There are three (3) senatorial zones in Kaduna State: Kaduna North, Kaduna Central, and Kaduna South Senatorial Zones. There are 1, 692 healthcare facilities; $40.2 \%$ being of the private sector; $3.2 \%$ secondary healthcare; $0.3 \%$ tertiary healthcare [11]. The major public health facility from each of the three senatorial zones was selected, plus an extra facility from the Kaduna Central zone which has the most 
population and is where the state capital is located at, thus making a total of four public health facilities for the study (two secondary and two tertiary health facilities).

Study tool. A questionnaire developed by Aljadhey et al. [6] was adopted for use in this study. The first section of the questionnaire contained questions on demographic information on location of hospital, hospital type and category, which best describes the hospital based on bed size. The second section was divided into seven (7) parts, which contained a total of 44 questions. Part 1 contained four (4) questions on medication safety committee and error reporting systems. The second part contained seven (7) questions focused on look-alike sound-alike (LASA) medications as they relate to error prevention. Questions in this part include availability of list of LASA medications, and mechanism for reviewing LASA medications, storage of medications in the pharmacy. The third part contained three (3) questions, which asked about hospital policy on control of concentrated electrolyte solutions. In part four (4), there were ten (10) questions on practices during transition in care. These include whether there was a policy for updating medication list, whether pharmacists take medication histories and whether patients are given a list of discharge medications. Part five contained ten (10) questions, which were specific to the availability and use of information technology in patient care. Questions included whether there was electronic access to inpatient laboratory values, use of computer to enter prescriptions, and pharmacy computer screening for drugto-drug allergy. Part six and seven of the survey asked about availability of drug information resources and implementation of other medication safety practices respectively. These practices included availability of error prone abbreviations, implementation of unit dose dispensing system and whether pharmacy staff receive baseline competency evaluation.

Data collection. The questionnaire was pretested in two facilities before the main study to test for local acceptability. Data collection took place from October 2018 to November 2018 in the form of a face-to-face structured interview that lasted 30 to 45 minutes with the Head of Pharmacy department of each facility. However, in one facility, the interview was conducted over a telephone call. Prior to data collection, a convenient time and location was agreed with each respondent for the questionnaire administration. Each aspect of the questionnaire was explained to the respondents and a written consent obtained (except in one case of verbal consent). Participants were asked to answer 'yes' or 'no' to each question, but were also allowed to give further reasons for their response.

Data analysis. Responses from the participants from the four health facilities were retrieved directly from the questionnaire based on a 'yes' or 'no' response to a question.

Ethical approval. This was obtained from Kaduna State Ministry of Health (MOH/ADM/744/VOL.1/499), Ahmadu Bello University Teaching Hospital (ABUTHZ/HREC/D21/2018) and Barau Dikko Teaching Hospital, Kaduna (1800011).

\section{RESULTS}

Medication safety practices in four public health facilities were assessed. Two of the facilities have a capacity of 100- 299 beds, while one was 300- 499 beds and the fourth was 500 beds and over (Table 1). Only one facility had a medication safety committee, only one facility has a paper error reporting system and none of the facilities had an electronic error reporting system (Table 2). None of the facilities had a list of look-alike, 
sound- alike (LASA) medications and none of the facilities provided healthcare providers education on LASA medications. Two facilities had concentrated electrolytes available in floor stock. None of the facilities uses 'high risk' warning label on diluted electrolyte solutions. Only one facility engages in taking drug history during care transitions by a pharmacist, but none of the facilities has a policy for updating patients' medication list. Regarding information technology (IT), only one of the facilities utilises information technology structures. However, it has no access to inpatient laboratory results and does not use medication bar coding. Although the pharmacy uses computer to enter prescriptions, there is no provision for screening for drug allergy. Also, body weight is not a required field. None of the facilities has drug information resources provided in all patient care areas and only one facility has computerized drug information resources available in the pharmacy. All four facilities have controlled drug formulary. However, none has a list of error prone abbreviations and none provides pharmacy staff baseline competency evaluation.

\section{DISCUSSION}

This study assessed the presence of basic medication safety practices and found out that many of these practices are not implemented, which provides room for improvements. Only one of the facilities had a medication safety committee. However, the committee is non-functional and is under the drug and therapeutics committee of the hospital. In addition, although there is a role stated of a medication safety officer in the committee, this has not been implemented as well. In a similar larger study in Saudi Arabia
[6], it was reported that only $30 \%$ of the hospitals had a medication safety committee, and $9 \%$ of hospitals had a medication safety officer. Another study, which assessed basic medication safety practices in 41 hospitals in Khartoum State, Sudan, reported similar findings of non-presence of medication safety committee and safety officers where only $5 \%$ of the hospitals had a medication safety committee, and none of the hospitals had a medication safety officer [12]. Reports from other countries have stated the beneficial roles of medication safety officers. The American Society of Health-system Pharmacists (ASHP) stated that having a medication safety officer is important for a medication safety programme to succeed and that the medication safety officer sets a vision and direction, identifies opportunities to improve the medication-use system, and lead implementation of error- prevention strategies [13].

Concerning error-reporting systems, only one facility has a paper based errorreporting system, which is mostly in the form of pharmacovigilance for reporting of adverse drug reactions (ADRs). However, the respondents noted that error reporting is not a common phenomenon in their healthcare settings, as it has not been inculcated in the culture of healthcare organisations. This is mostly due to healthcare providers believing reporting an error could result to punitive measures and blame may be apportioned. However, it is important to educate healthcare providers that the fundamental role of a patient safety reporting system is to enhance patient safety by learning from failures of the health-care system and that reporting plays a key role in detecting patient safety problems.

Table 1: General characteristics of the selected public health facilities

\begin{tabular}{lllll}
\hline & Hospital A & Hospital B & Hospital C & Hospital D \\
\hline Senatorial Zone & Kaduna North & Kaduna Central & Kaduna Central & Kaduna South \\
Type of hospital & Tertiary hospital & Tertiary hospital & Secondary hospital & Secondary hospital \\
Number of beds & 500 beds and over & 100-299 beds & 100-299 beds & 300- 499 beds \\
\hline
\end{tabular}


Table 2: Responses from the selected public health facilities on medication safety practices

\begin{tabular}{|c|c|c|c|c|c|}
\hline & \multicolumn{4}{|c|}{ Health facilities } \\
\hline & & A & B & $\mathrm{C}$ & $\mathrm{D}$ \\
\hline & PART 1: Medication Safety Committee and error reporting systems & & & & \\
\hline 1 & Is there a medication safety committee in this hospital? & Yes & No & No & No \\
\hline 2 & Is there a medication safety director? & Yes & No & No & No \\
\hline 3 & Is any a paper- based error reporting system used? & Yes & No & No & No \\
\hline \multirow[t]{2}{*}{4} & Is an electronic error reporting system implemented? & No & No & No & No \\
\hline & PART 2: Look-alike Sound-alike medications & & & & \\
\hline 5 & Is there available a list of (LASA) medications? & No & No & No & No \\
\hline 6 & Is there a mechanism for reviewing LASA medications? & No & No & No & No \\
\hline 7 & Is there a mechanism to prevent LASA medications? & No & No & No & No \\
\hline 8 & Are healthcare providers given education on LASA medications? & No & No & No & No \\
\hline 9 & Are medications stored in pharmacy alphabetically? & No & No & No & No \\
\hline 10 & Does diagnosis field exists in the prescription or drug order? & No & No & No & No \\
\hline \multirow[t]{2}{*}{11} & Are both brand and generic names included on medication labels? & No & No & No & No \\
\hline & PART 3: Control of concentrated electrolyte solution & & & & \\
\hline 12 & Are concentrated electrolytes found on ward (floor) stock? & Yes & No & Yes & No \\
\hline 13 & $\begin{array}{l}\text { Does a second person verify final concentrations of parenteral electrolyte solutions } \\
\text { including calculations? }\end{array}$ & No & No & No & No \\
\hline \multirow[t]{2}{*}{14} & Are high-risk warning labels used on diluted electrolyte solution? & No & No & No & No \\
\hline & PART 4: Transition in care & & & & \\
\hline 15 & Is a new order required with patient admission or transfer? & Yes & Yes & Yes & Yes \\
\hline 16 & Are orders stating "resume the same medications" accepted? & No & No & Yes & Yes \\
\hline 17 & Does a policy for updating medication list exist? & No & No & No & No \\
\hline 18 & Is a complete drug history taken during care transitions? & Yes & No & No & No \\
\hline 19 & Does a pharmacist take medication history? & Yes & No & No & No \\
\hline 20 & Are patients' current medications list put in consistent highly visible location? & Yes & Yes & No & No \\
\hline 21 & Are there written policies and procedures to list and update the medication list? & No & No & No & No \\
\hline 22 & Is a patient's current medication list updated with new physician orders? & Yes & No & No & No \\
\hline 23 & Are patients given a list of discharge medications? & Yes & Yes & Yes & No \\
\hline \multirow[t]{2}{*}{24} & Are health care professionals educated on procedures for reconciling medications? & Yes & No & No & No \\
\hline & PART 5: Information Technology & & & & \\
\hline 25 & Is there electronic access to inpatient laboratory values? & No & No & No & No \\
\hline 26 & Is there use of medication bar coding? & No & No & No & No \\
\hline 27 & Is there electronic medication administration record? & Yes & No & No & No \\
\hline 28 & Does pharmacy use computer to enter prescription? & Yes & No & No & No \\
\hline 29 & Is patient allergy history required to enter an order? & Yes & No & No & No \\
\hline 30 & Is there provision for drug allergy verification? & No & No & No & No \\
\hline 31 & Does the pharmacy computer screens drug for drug allergy? & No & No & No & No \\
\hline 32 & Is an allergy list clearly visible on all pages of medication administration records? & No & No & No & No \\
\hline 33 & Is the computer directly interfaced with the laboratory? & No & No & No & No \\
\hline \multirow[t]{2}{*}{34} & Is body weight a required field? & No & No & No & No \\
\hline & PART 6: Drug Information & & & & \\
\hline 35 & Are drug information resources provided in all patient $\mathrm{c}$ & No & No & No & No \\
\hline \multirow[t]{2}{*}{36} & Are computerized drug information resources available in the pharmacy? & No & Yes & No & No \\
\hline & PART 7: Other Medication Safety Practices & & & & \\
\hline 37 & Is renal or hepatic dosage adjustment for relevant patients done? & Yes & No & No & No \\
\hline 38 & Is there a maximum dose for high alert drug? & No & No & No & No \\
\hline 39 & Is there a controlled drug formulary system? & Yes & Yes & Yes & Yes \\
\hline 40 & Is a list of error prone abbreviations available? & No & No & No & No \\
\hline 41 & Has unit dose system been implemented? & Yes & No & No & No \\
\hline 42 & Are medications brought from home by patient not used? & Yes & Yes & No & Yes \\
\hline 43 & Are discontinued medications removed from patient supplies in a timely manner? & Yes & Yes & Yes & Yes \\
\hline 44 & Do pharmacy staff receive baseline competency evaluation? & No & No & No & No \\
\hline
\end{tabular}


Regarding practices related to lookalike, sound-alike (LASA) medications, this appeared quite unfamiliar to all respondents and none of the facilities has a list of LASA medications nor educate staff on LASA medications. Reports have shown that existence of confusing drug names is one of the most common causes of medication errors worldwide [14]. It is important for healthcare organisations to put great considerations concerning LASA medications to avoid errors. Provision of list of LASA medications may be a simple way of intervention by educating staff about LASA medications.

Only one of the facilities involves pharmacist in taking patient history. Studies have shown that involving pharmacists in taking medication histories through medication reconciliation activities helps in reducing errors $[15,16]$ and also involvement of pharmacy staff in medication reconciliation activities results in obtaining an accurate medication history more often than when pharmacy staff are not involved [17]. The Institute for Healthcare Improvement (IHI) defines medication reconciliation as "the process of creating the most accurate list possible of all medications a patient is taking - including drug name, dosage, frequency, and route - and comparing that list against the physician's admission, transfer, and/or discharge orders, with the goal of providing correct medications to the patient at all transition points within the hospital" [18]. Medication reconciliation has been recognised as a major intervention that tackles the problems of medication discrepancies and subsequent patient harm at transitions in care [18]. However, this is best achieved when there is a sufficient number of pharmacists and pharmacists are in direct patient care by a having a presence in patient wards. At present, most healthcare facilities in Kaduna State have pharmacists only playing dispensing roles and do not get the opportunity to play other roles in direct patient care. Optimising medication reconciliation is important in ensuring patient safety at low cost [19]. Although it can increase the work demand for healthcare professionals, involvement of more than one type of professional (particularly pharmacists and physicians) can enhance the quality of the overall process.

Information technology (IT) facilities were also lacking in the health facilities in this study. Although the respondents stated that there have been efforts to set up these facilities, there needs to be availability of constant electricity and internet infrastructure for proper adoption of the IT tools. Studies have shown the relevance of IT interventions in improving medication safety $[1,6,20,21]$. It has been shown that the use of computerised physician order entry reduced medication error rates by $55 \%$ [1] and another study showed that dispensing errors were minimised by $31 \%$ by the use of bar coding [20]. In addition, IT interventions have been shown to be successful in reducing medication errors especially if the IT intervention is accompanied by collaboration between pharmacists and physicians [21]. However, Aljadhey et al. [6] pointed out that as IT interventions may be costly, basic interventions identified through adoption of other practices could be implemented first. This is particularly important for developing countries where implementation of IT interventions could prove costly.

The Institute for Safe Medication Practices (ISMP) stated that selecting the best error-reduction strategy is not easy [22]. ISMP further described these strategies in three levels with 'High-leverage strategies' (such as automation and computerisation) focus on the system hazards and are most effective because they can eliminate the risk of errors and associated harm. 'Mediumleverage strategies' (such as reminders and checklists; warnings, alerts, and alarms; and patient counselling) do not eliminate hazards 
but reduce the likelihood of errors or minimise harm. They are relatively easy to implement but may need periodic updating and reinforcement to maintain knowledge and the currency of the process or product. 'Lowleverage strategies' (such as protocols, guidelines, education and training) are often easy and quick to implement but need constant updating and reinforcement to maintain knowledge. They aim to improve human performance and are more effective when combined with other medium or high leverage strategies. Importantly, medication errors, like other types of patient safety incidents, usually arise from human factors and poorly designed healthcare products and systems rather than the individual performance of a single practitioner. Ensuring safe medication practice requires an understanding of human factors (the reasons why humans make errors), and that healthcare products and systems should be designed to minimise the risks of errors harming patients [17].

As with other studies $[6,12]$ that assessed medication safety practices in line with recommendations made by ISMP and WHO, there is need to enforce laws and regulations by policy makers and Ministry of Health making implementation of medication safety practices mandatory to ensure patient safety. This will also require the efforts of all stakeholders involved: the healthcare workers, administrators, patients and even caregivers.

Acknowledgement. The authors would like to acknowledge the participants who took time out of their busy schedule to take part in the study.

\section{REFERENCES}

1. Bates DW, Leape LL, Cullen DJ, et al. Effect of Computerized Physician Order Entry and a Team Intervention on Prevention of Serious Medication Errors. JAMA. 1998; 280: 1311-6.

2. World Health Organisation. Patient Safety. 2011. Retrieved 21 March 2018, from://www.who.int/patientsafety/about/en/ Accessed 21 March 2018

3. Kaushal, R, Bates, DW, Landrigan, C, McKenna, KJ, Clapp, MD, Federico, F, and Goldmann, DA. Medication errors and adverse drug events in pediatric inpatients. JAMA. 2001; 285(16), 21142120 .

4. World Health Organisation. Media centre. 2017. Retrieved 9 May 2017, from: http://www.who.int/mediacentre/news/releases/2017/ medication-related-errors/en/

5. World Health Organisation. Medication Without Harm. 2017. Retrieved 9 July 2019, from https://www.who.int/patientsafety/medicationsafety/medication-without-harm-brochure/en/

6. Aljadhey H, Alhossan A, Alburikan K, Adam M, Murray MD and Bates DW. Medication safety practices in hospitals: A national survey in Saudi Arabia. Saudi pharmaceutical journal: SPJ: the official publication of the Saudi Pharmaceutical Society. 2013; 21: 159-64.

7. Cohen M, Kimmel N, Benage M, et al. Medication safety program reduces adverse drug events in a community hospital. BMJ Quality \& Safety. 2005; 14: 169-74.

8. Gleason KM, Groszek JM, Sullivan C, Rooney D, Barnard $\mathrm{C}$ and Noskin GA. Reconciliation of discrepancies in medication histories and admission orders of newly hospitalized patients. American Journal of Health-System Pharmacy. 2004; 61: 168995.

9. Strunk LB, Matson AW and Steinke D. Impact of a pharmacist on medication reconciliation on patient admission to a veterans affairs medical center. Hospital Pharmacy. 2008; 43: 643-9.

10. World Health Organisation. World Alliance for Patient Safety. 2011. Retrieved 10 March 2018, from: http://www.who.int/patientsafety/worldalliance/en/ Accessed 10 March 2018.

11. Kaduna State Government. Strategic Health Development Plan (2010- 2015). 2010. Kaduna.

12. Abdulrahman S, Mahmoud M, Ibrahim A, and Aljadhey H. An assessment of the basic medication safety practices in Khartoum State hospitals. European Journal of Hospital Pharmacy. 2016; 23. doi: 10.1136/ejhpharm-2016-000885

13. American Society of Health-System Pharmacists (ASHP). Medication safety. 2018 Retrieved 20 May 2019, from https://www.ashp.org/advocacy-andissues/key-issues/medication-safety 
14. World Health Organisation (WHO). The nine patient safety solutions, 2007. World Health Organisation, 2011. Retrieved 03 March 2017, from: http://www.who.int/patientsafety/events/07/02_05_20 07/en/

15. Abuyassin, B, Aljadhey, H, and Al-Sultan, M. Accuracy of the medication history at admission to hospital in Saudi Arabia. Saudi Pharmaceutical Journal. 2011; 19, 263-267

16. Strunk, LB, Matson, AW, and Steinke, D. Impact of a pharmacist on medication reconciliation on patient admission to a veterans affairs medical center. Hospital Pharmacy. 2008; 43(8), 643-649

17. Mueller, SK, Sponsler, KC, Kripalani, S, and Schnipper, JL. Hospital-based medication reconciliation practices: a systematic review. Archives of Internal Medicine. 2012; 172(14), 10571069. doi: 10.1001/archinternmed.2012.2246

18. Institute for Healthcare Improvement. Medication reconciliation review. Retrieved 4 May 2017, from http://www.ihi.org/resources/pages/tools/Medication $\underline{\text { ReconciliationReview.aspx }}$
19. Meguerditchian, AN, Krotneva, S, Reidel, K, Huang, A, and Tamblyn, R. Medication reconciliation at admission and discharge: a time and motion study. BMC Health Services Research. 2013; 13, 485-485. doi: 10.1186/1472-6963-13-485

20. Poon, EG, Cina, JL, Churchill, W, Patel, N, Featherstone, E, Rothschild, JM, . . . Gandhi, TK. Medication dispensing errors and potential adverse drug events before and after implementing bar code technology in the pharmacy. Annals of internal medicine. 2006; 145(6), 426-434.

21. Lainer, M, Mann, E, and Sönnichsen, A. Information technology interventions to improve medication safety in primary care: a systematic review. International Journal for Quality in Health Care. 2013; 25(5), 590-598. doi: 10.1093/intqhe/mzt043

22. Institute for Safe Medication Practices (ISMP). 2011. Retrieved 3 March 2017, from: <http://www.ismp.org/selfassessments/default.asp>. Accessed 03 March 2017 